\title{
Fecal Microbiota Transplantation in Patients With Recurrent Clostridium difficile Infection: A Four-Year Single-Center Retrospective Review
}

\author{
Muhammad Farhan Ashraf ${ }^{\mathrm{a}, \mathrm{c}} \mathbb{(} \mathrm{C}$, Omar Tageldin $^{\mathrm{a}}$, Yousef Nassar ${ }^{\mathrm{b}}$, Asra Batool $^{\mathrm{b}}$
}

\begin{abstract}
Background: Clostridium difficile infection (CDI) is a common cause of hospital and community-acquired diarrhea with an annual incidence of 453,000 cases in the USA. The white race, female gender, and age over 65 years are known risk factors. Recurrence of CDI is a major problem in patients taking antibiotics for prolonged periods. These patients are observed to have reduced diversity of the intestinal microbiome. Fecal microbiota transplantation (FMT) can restore the healthy flora in the gut, thus breaking the cycle of recurrent infection. Our study aimed to analyze the efficacy of FMT and the recurrence of CDI after FMT. We also aimed to investigate the effects of comorbidities on the outcome of FMT.
\end{abstract}

Methods: After obtaining approval from the institutional review board, we included 64 patients who had received FMT at our institution from October 2015 to November 2019. All the patients over 16 years of age in both inpatient and outpatient settings were included. Patients under 16 years of age and patients treated without FMT were excluded. Frozen stool from a standardized stool bank (OpenBiome) was used. The thawed specimen was instilled into the terminal ileum or the cecum. Patients were followed up for the next 1 year for analysis of improvement in symptoms, recurrence, and repeat FMT.

Results: On the 2-months follow-up, 75\% of patients reported symptomatic improvement, $15.6 \%$ reported no improvement while $9.4 \%$ did not follow up. Twenty-six (40.6\%) patients had CDI recurrence in the following year; and $69.2 \%$ of patients with recurrence underwent a repeat FMT. There was no statistically significant correlation between CDI recurrence and the age $(\mathrm{P}$ value $=0.68)$, gender $(\mathrm{P}$ value $=0.61$ ), previous use of proton pump inhibitors (PPIs, P value $=0.11$ ) or antibiotics ( $\mathrm{P}$ value $=0.45)$. There was a statistically significant correlation noted with the use of immunosuppressants and recurrence $(\mathrm{P}$ value $=0.04)$.

Manuscript submitted June 15, 2021, accepted July 13, 2021

Published online July 28, 2021

aDepartment of Internal Medicine, Albany Medical Center, Albany, NY, USA bDepartment of Gastroenterology, Albany Medical Center, Albany, NY, USA ${ }^{\mathrm{c} C o r r e s p o n d i n g ~ A u t h o r: ~ M u h a m m a d ~ F a r h a n ~ A s h r a f, ~ D e p a r t m e n t ~ o f ~ I n t e r n a l ~}$ Medicine, Albany Medical Center, Albany, NY, USA.

Email: ashrafm1@amc.edu

doi: https://doi.org/10.14740/gr1436
Conclusions: FMT is a successful treatment modality for refractory and recurrent CDI. Repeat treatments can be beneficial if there is a lack of initial response. Being immunosuppressed with medications is associated with the risk of recurrence.

Keywords: Fecal microbiota transplant; Clostridium difficile infection; Frozen stool sample; Colonoscopy

\section{Introduction}

Clostridium difficile infection (CDI) is a common cause of hospital and community-acquired diarrhea with an annual incidence of 453,000 cases in the USA. Every year 29,000 people die of CDI in their first 30 days after diagnosis in the USA [1]. A higher incidence of CDI has been reported in the white race, female gender, and 65 or older population [2]. The recurrence of CDI is a challenging problem in patients taking antibiotics. These patients are observed to have reduced diversity of the intestinal microbiome resulting in recurrent CDI. Fecal microbiota transplantation (FMT) from healthy individuals to these patients can restore healthy flora in the gut, thus breaking the cycle of recurrent infection [3].

Historically, coprophagy (ingestion of fecal material) has been observed in many animal species likely contributing to the prevention of colonization of pathogens $[4,5]$. The earliest use of fecal material in human history for the treatment of diarrhea dated to the fourth century in China. Later records of ancient gastrointestinal remedies refer to fecal products as "yellow soup" or "golden syrup" for the treatment of several gastrointestinal and systemic diseases such as diarrhea, malaria, severe food poisoning, and fever [6, 7]. In modern medicine, Eiseman et al in the year 1958 successfully treated four patients with pseudomembranous colitis through fecal enemas prepared from healthy donor stool [8]. Currently, FMT is mostly used for the treatment of recurrent Clostridium difficile colitis. The Infectious Disease Society of America (IDSA) in its updated clinical practice guidelines for the treatment of CDI strongly recommends FMT for patients with multiple recurrences of CDI despite appropriate antibiotic treatments [9]. Both frozen and fresh microbiota can be used for transplant. The rates of clinical resolution among frozen and fresh FMT were comparable ( $75 \%$ versus $70 \%)$ in a randomized clinical 
trial including 219 patients with the added advantage of using frozen microbiome stool banks for better screening [10]. In recent years there has been much debate regarding the safety of FMT which mainly stems from the idea of potentially introducing harmful bacteria in recipients through donor feces. Although, FMT is Food and Drug Administration (FDA) approved only as an experimental treatment for recurrent CDI, yet it has multiple potential applications in both intestinal and extra-intestinal disorders, some of them being inflammatory bowel disease (IBD), irritable bowel syndrome, and metabolic syndrome.

The purpose of our study was to analyze the efficacy of FMT in decreasing the recurrence of CDI and assess comorbidities and their relationship with the outcomes in this patient population.

\section{Materials and Methods}

\section{Study design and sample}

After institutional review board (IRB) approval, a retrospective electronic medical record chart review was performed on 64 patients in the institutional FMT registry who underwent FMT between October 1, 2015 and November 20, 2019 at Albany Medical Center, Department of Gastroenterology. During these 4 years, seven cases of FMT were performed in 2015, 21 cases of FMT were performed in 2016, 16 cases were performed in 2017, 17 cases were performed in 2018 while 26 cases were performed in 2019. Data were collected from electronic medical records of the patient visits to the gastroenterology clinic, endoscopy reports, and hospital admissions. Patients were followed up to 1 year after FMT. The patient population variables examined were age, gender, history of antibiotic use before FMT, prolonged proton pump inhibitor (PPI) use (defined by more than 1 month of PPI use), inpatient versus outpatient location, in-house versus outside referral, use of immunosuppressants, colonoscopy findings, time of stopping antibiotics before FMT, number of recurrences of CDI before FMT, number of FMT done in past, time to symptom improvement, duration of hospital stay, subsequent recurrence, subsequent FMT, and adverse events after the procedure. Besides, data on comorbidities such as immunocompromised state, diabetes, and hypertension were collected. Other variables included were the use of vancomycin, metronidazole, fidaxomicin, or all three before FMT. Recurrence was defined as relapse of CDI symptoms 2 weeks after the procedure. A successful FMT was defined as more than 50\% improvement in the number of episodes of diarrhea after FMT. Both hospital and follow-up clinical records were reviewed to determine how many bowel movements patients were having before and after FMT. Patients were followed up for 2 months for symptomatic improvement. Patients with less than $50 \%$ improvement or no improvement in 2 months following FMT were deemed a failure.

\section{Donor stool preparation and delivery}

Pre-screened donor stool was obtained from the stool bank (Open Biome) in the form of frozen specimens. Both frozen and fresh stool preparation is used for FMT, but since our facility uses only frozen stool, fresh stool samples were not used in our study. Preparation of stool specimens for transplant was done in the endoscopy suite before administration. Colonoscopy was performed using minimum air insufflation and the specimen was instilled into the terminal ileum or the cecum. Patients were advised to remain supine after the procedure for as long as possible to retain the transplanted stool.

\section{Outcomes}

Greater than 50\% improvement in symptoms within 2 months after the fecal transplant was the primary outcome of the study. Secondary outcomes included recurrence of CDI after FMT and the need for subsequent FMT. After the transplant, patients had follow-up appointments setup with our gastroenterology clinic in 6 - 8 weeks to evaluate for symptom improvement. Telephone calls were also made by staff during this period to evaluate response to transplant and documented in electronic medical records. Questions were asked regarding adverse effects of the procedure, consistency of stools, and frequency of bowel movements. The patients were not tested for CDI after FMT to determine the resolution of infection as per guidelines to prevent falsepositive results [11]. Patients experiencing persistent or recurrent symptoms after FMT were evaluated for a repeat procedure.

\section{Statistical analysis}

Statistical analysis was performed using IBM SPSS (Version 22). Frequency tables, Chi-square tests, Fisher's exact test, and cross-tabulations were used to analyze any correlation between variables under investigation and outcomes of the study. A P value of $<0.05$ was determined to be statistically significant.

\section{Ethical compliance with human study}

This study was conducted in compliance with the ethical standards of the responsible institution on human subjects as well as with the Helsinki Declaration.

\section{Results}

\section{Patient population}

The study included 64 patients. Their ages ranged from 16 to 91 years. Thirty-seven patients $(57.8 \%)$ were female, and 27 patients $(42.2 \%)$ were male.

\section{Role of antibiotic use}

About $59.4 \%$ of patients had a history of antibiotic use before CDI $(n=38)$. Forty-six patients $(71.9 \%)$ had antibiotics for CDI stopped $>48 \mathrm{~h}$ before the procedure, while 18 patients $(28.1 \%)$ 
Table 1. Demographic Variables $(N=64)$

\begin{tabular}{|c|c|}
\hline & $\mathbf{N}(\%)$ \\
\hline \multicolumn{2}{|l|}{ Gender } \\
\hline Male & $27(42.2 \%)$ \\
\hline Female & $37(57.8 \%)$ \\
\hline \multicolumn{2}{|l|}{ Referral } \\
\hline In-house & $55(85.9 \%)$ \\
\hline Outside & $9(14.1 \%)$ \\
\hline Inpatient & $37(57.8 \%)$ \\
\hline Outpatient & $27(42.2 \%)$ \\
\hline \multicolumn{2}{|l|}{ Comorbidities and risk factors } \\
\hline History of PPI use & $27(42.2 \%)$ \\
\hline Antibiotics used before CDI & $38(59.4 \%)$ \\
\hline Use of immunosuppressants & $12(18.8 \%)$ \\
\hline Diabetes & $17(26.6 \%)$ \\
\hline Hypertension & $38(59.4 \%)$ \\
\hline Immunocompromised state including IBD & $25(39.1 \%)$ \\
\hline \multicolumn{2}{|c|}{ Antibiotics used for the treatment of CDI before FMT } \\
\hline Vancomycin alone & $14(21.8 \%)$ \\
\hline Fidaxomicin alone & $0(0 \%)$ \\
\hline Metronidazole alone & $2(3.1 \%)$ \\
\hline Vancomycin + metronidazole & $16(25.0 \%)$ \\
\hline Vancomycin + fidaxomicin & $10(15.6 \%)$ \\
\hline Fidaxomicin + metronidazole & $0(0 \%)$ \\
\hline No antibiotic therapies & $1(1.5 \%)$ \\
\hline All three & $21(42.1 \%)$ \\
\hline Antibiotics stopped $>48 \mathrm{~h}$ before FMT & $46(71.9 \%)$ \\
\hline Adverse events from FMT & $12(18.8 \%)$ \\
\hline Abnormal colonoscopy findings & $39(60.9 \%)$ \\
\hline
\end{tabular}

PPI: proton pump inhibitors; CDI: Clostridium difficile infection; IBD: inflammatory bowel disease; FMT: fecal microbiota transplant.

had antibiotics stopped $<48 \mathrm{~h}$ before the procedure. Vancomycin was the most common antibiotic used in these patients for CDI before FMT, with $61(95.3 \%)$ patients using vancomycin either in combination or monotherapy. Fourteen patients $(21.1 \%)$ used vancomycin alone while 16 patients $(25 \%)$ used a combination of vancomycin and metronidazole, 10 patients $(15.6 \%)$ used a combination of vancomycin and fidaxomicin. Metronidazole monotherapy was observed in two patients (3.1\%). Monotherapy with fidaxomicin or a combination of fidaxomicin and metronidazole was not observed in any patients. Twenty-one patients $(42.1 \%)$ had used all three antibiotics to treat CDI before FMT.

\section{Other variables}

Twenty-seven patients (42.2\%) had a history of prolonged PPI use (more than 1 month), while 37 patients $(57.8 \%)$ did not
Table 2. Primary and Secondary Outcomes $(N=64)$

\begin{tabular}{ll}
\hline & N (\%) \\
\hline Improvement in 2 months & $48(75.0 \%)$ \\
FMT failure & $10(15.6 \%)$ \\
Patients lost to follow-up & $6(9.4 \%)$ \\
Recurrence of CDI after 2 months & $26(40.0 \%)$ \\
\hline
\end{tabular}

CDI: Clostridium difficile infection; FMT: fecal microbiota transplant.

have a history of PPI use. Twelve patients (18.8\%) were on immunosuppressant therapy before FMT (Table 1). Patients were either referred for FMT from our hospital's gastroenterology clinic/inpatient facility (in-house referrals) or outside clinics (outside referrals). Fifty-five patients $(84.9 \%)$ had an in-house referral and the success rate of FMT in these patients (defined by more than $50 \%$ improvement in 2 months) was $78.2 \%$. Both Chi-square and Fisher's exact test showed a significant correlation between referral type and the result of FMT $(\mathrm{P}=$ 0.002 ). Only one variable, referral type had a statistically significant correlation with the primary outcome (improvement in 2 months). This was likely due to better follow-up in patients with in-house referrals as four out of six patients who were lost to follow-up were referred from outside facilities.

\section{Primary outcome}

The overall success rate of FMT in our study was 75\% with 48 out of 64 patients showing improvement in the first 2 months after FMT (Table 2). Six patients $(9.4 \%)$ were lost to follow-up of which four patients were outside referrals. Ten patients $(15.6 \%)$ reported no improvement and were deemed treatment failure. Among these 10 patients who had failed treatment, four patients underwent a repeat FMT with improvement in symptoms of which three patients improved, while one failed repeat FMT requiring a colectomy. Among the remaining six patients who had failed treatment but did not receive repeat FMT, three patients died from various etiologies before getting the repeat FMT, two patients showed improvement after the first 2 months while one patient underwent colectomy. Thirty-nine $(60.9 \%)$ patients had abnormal colonoscopy findings with pancolitis being the most common (18.8\%, Fig. 1). Twelve patients (18.8\%) reported adverse events after FMT. The most reported adverse event was abdominal pain/cramping (10.9\%). Other adverse events included worsening diarrhea in three $(4.6 \%)$ patients, bloating in one $(1.6 \%)$ patient, and weight gain in one $(1.6 \%)$ patient.

\section{Secondary outcomes}

Secondary outcomes in our study were the rate of CDI recurrence and the need for repeat FMT after the initial procedure. CDI recurrence was defined as a positive Clostridium difficile polymerase chain reaction (PCR) test in symptomatic patients at least 2 months after initial FMT. CDI recurrence was reported in 26 patients (40.6\%). On average, CDI recurrence occurred 113 days after the initial FMT. Nine patients $(34.6 \%)$ used antibiotics 


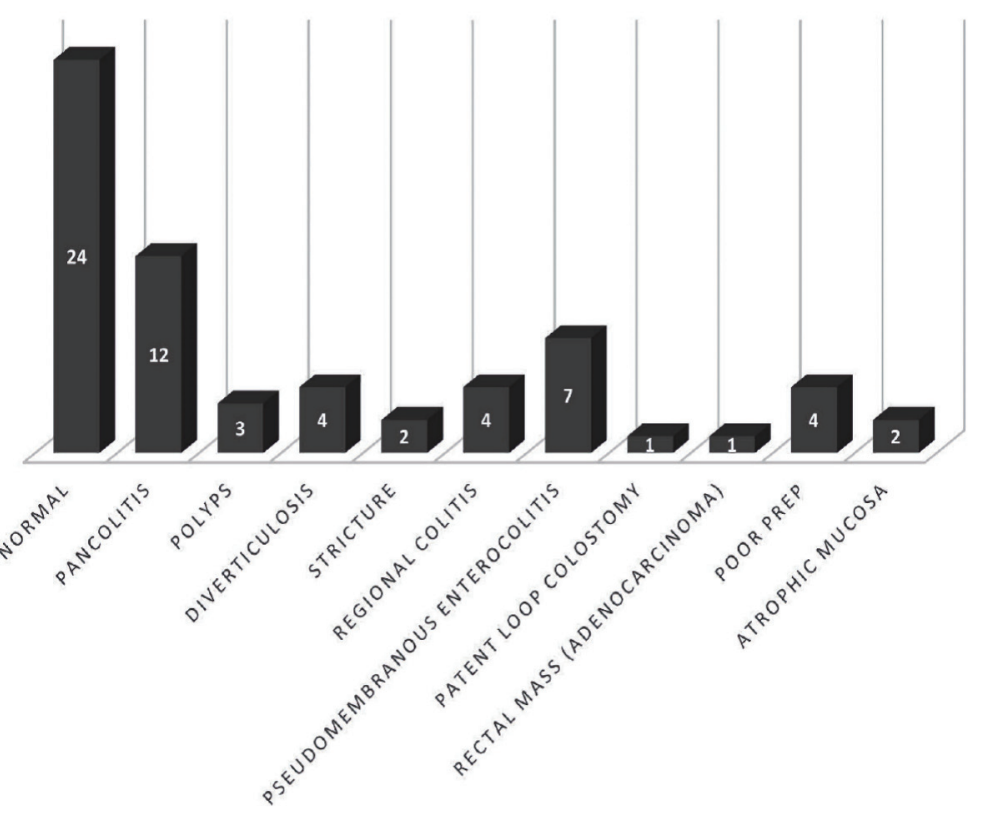

Figure 1. Colonoscopy findings.

for reasons other than treatment of CDI before recurrence. Eight patients $(30.8 \%)$ were on immune suppressants for either IBD or to prevent transplant rejection while two patients had IBD but were not taking any immune suppressants. Among these eight patients on immune suppressants, six had IBD, one patient was on chemotherapy for leukemia while one patient was a renal transplant recipient maintained on tacrolimus. Crohn's disease and ulcerative colitis were observed in three patients each. Being on immunosuppressant therapy had a statistically significant relationship with recurrence of CDI $(\mathrm{P}=0.04$, Table 3$)$. The ma-

Table 3. Success and Recurrence Rates in Different Demographic Variables and Risk Factors

\begin{tabular}{|c|c|c|c|c|c|}
\hline Demographic variables & $\mathbf{N}$ & Success $^{\mathrm{a}}$ & P value & Recurrence & P value \\
\hline \multicolumn{6}{|l|}{ Gender } \\
\hline Male & 27 & $23(85.3 \%)$ & & $10(37.0 \%)$ & \\
\hline \multicolumn{6}{|l|}{ Referral } \\
\hline In-house & 55 & $43(78.2 \%)$ & & $21(38.2 \%)$ & \\
\hline Outpatient & 27 & $18(66.7 \%)$ & 0.09 & $9(33.3 \%)$ & 0.31 \\
\hline \multicolumn{6}{|l|}{ Comorbidities and risk factors } \\
\hline History of PPI use & 27 & $21(77.8 \%)$ & 0.66 & $14(51.9 \%)$ & 0.11 \\
\hline Antibiotics used before CDI & 38 & $27(71.1 \%)$ & 0.05 & $14(36.8 \%)$ & 0.45 \\
\hline Immunocompromised state & 25 & $19(76 \%)$ & 0.72 & $11(44.0 \%)$ & 0.66 \\
\hline Antibiotics stopped $>48 \mathrm{~h}$ before FMT & 46 & $34(73.9 \%)$ & 0.90 & $17(37.0 \%)$ & 0.19 \\
\hline Adverse events from FMT & 12 & $7(58.3 \%)$ & 0.17 & $3(25.0 \%)$ & 0.22 \\
\hline Abnormal colonoscopy findings & 37 & $26(70.3 \%)$ & 0.53 & $15(40.5 \%)$ & 0.62 \\
\hline
\end{tabular}

aSuccess was defined as more than $50 \%$ improvement of symptoms in 2 months. Values were shown as n (\%). CDI: Clostridium difficile infection; FMT: fecal microbiota transplant; PPI: proton pump inhibitor. 
Table 4. Characteristics of Patients With CDI Recurrence (N $=26$ )

\begin{tabular}{ll}
\hline & N (\%) \\
\hline Antibiotic use before CDI recurrence & $9(34.6 \%)$ \\
Immunosuppressant use before recurrence & $8(30.7 \%)$ \\
Crohn's disease & $3(11.5 \%)$ \\
Ulcerative colitis & $3(11.5 \%)$ \\
Organ transplant recipients & $1(3 \%)$ \\
Treatment with repeat FMT & $18(69.2 \%)$ \\
Treatment with antibiotics & $7(26.9 \%)$ \\
\hline
\end{tabular}

CDI: Clostridium difficile infection; FMT: fecal microbiota transplant.

jority of these patients with CDI recurrence were treated with repeat FMT. Eighteen patients $(65.3 \%)$ with CDI recurrence were treated with repeat FMT while antibiotics were used to treat seven patients $(26.9 \%)$. One patient died before receiving a repeat FMT. Of the seven patients treated with antibiotics, vancomycin was used to treat five patients. These findings are summarized in Table 4. Interestingly, three patients who had received a successful repeat FMT after treatment failure during the first 2 months showed CDI recurrence in the next year and underwent a third FMT with $100 \%$ success. The success rate in patients undergoing two FMTs was $70.6 \%$ while a $100 \%$ success rate was observed in patients with three FMTs. The single patient who had four FMTs failed therapy finally underwent a total proctocolectomy.

\section{Discussion}

We conducted a single-center retrospective chart review for patients with recurrent CDI undergoing FMT between October 1, 2015 and November 20, 2019 at Albany Medical Center, Department of Gastroenterology. Our results with frozen fecal microbiota were comparable to other studies and showed a success rate of $75 \%$. About $10 \%$ of patients were lost to follow-up therefore actual success rate may be higher than $75 \%$. A cure rate as high as $91.9 \%$ has been reported in the literature [12]. Both fresh and frozen fecal microbiota can be used for this procedure with comparable results in both groups $[10,13]$. A randomized clinical trial by Lee et al [10] showed $70.3 \%$ and $75.0 \%$ clinical resolution in fresh versus frozen fecal samples. At our facility, only prescreened frozen specimens are used hence our study is based on frozen fecal microbiota.

The patients in our study had a variety of comorbidities such as hypertension, diabetes, immunosuppressed state, and PPI use. Our study noted that among the comorbidities studied, being on an immunosuppressant medication had a statistically significant association with recurrence. There was no statistically significant relationship between being on immunosuppressants and symptom resolution in these patients. In our study, patients on immunosuppressants had a $90.9 \%$ success rate. But on the other hand, these patients were also at risk for recurrence as mentioned above. A systematic review of 44 studies showed an $87 \%$ success rate in immunocompromised patients who underwent their first FMT, and the cure rate increased to $93 \%$ on subsequent FMTs [14]. A possible reason why being on immunosuppressant therapy had an association with recurrence and the need for repeat FMT is that these patients are more likely to be colonized with Clostridium difficile in general, and thus can have a higher recurrence rate. This makes being on immunosuppressants an independent risk factor for recurrent CDI as shown in a previous study [15]. In the past few years, multiple studies and meta-analysis have shown a significant relationship between PPI use and the risk of CDI while some studies have rejected this hypothesis [16-18]. Our study did not show a causal relationship between PPI use and recurrence of CDI.

In our tertiary referral center, there were more in-house than outside referrals. In-house referrals showed better outcomes. This can be explained by a higher loss of follow-up in outside referrals. Of the six patients lost to follow-up, four patients were referred from outside gastrointestinal clinics.

Although the inpatient population tends to be sicker and treatment is challenging, our study showed a success rate of $81 \%$ in inpatient versus $66.7 \%$ in outpatient cases. This can be explained by a higher number of inpatient cases (37 inpatients versus 27 outpatients) and a higher loss of follow-up in the outpatient population as described above. Also, patients with outpatient cases reported subjective changes in symptoms while the inpatient population had their number of daily bowel movements and other symptoms quantified and charted by nursing staff making this data more accurate.

Although FMT offers a higher cure rate, it does not come without its limitations. Our study showed that recurrence of CDI after FMT in the following years is still a major concern despite high symptom resolution rates. Three patients in our study died during the follow-up period due to various reasons. Only one patient died secondary to uncontrolled CDI. In a systemic review of 15 studies, the all-cause 30-day mortality in patients with CDI was seen in $15 \%$ of patients while mortality attributed to CDI in these patients ranged from $5.7 \%$ to $6.9 \%$ [19].

Our study had certain limitations including its retrospective design and small sample size. Since FMT is an innovative treatment option and only approved as an experimental treatment as per FDA, we only had 64 patients over 4 years in our study. To determine the generalizability of our results, larger sample size will be beneficial. Certain confounders can be missed in a small sample population. Despite being limited by our sample size, our study showed a cure rate of FMT comparable to available literature, and we were able to show a statistically significant relationship between the use of immunosuppressants and the recurrence of CDI.

Future studies on FMT should include a comparison of different FMT protocols used these days to help develop a standard FMT protocol [20]. Recently, questions have been raised regarding the safety of FMT. A standard FMT protocol with standardized screening for drug-resistant bacteria in donor stool can make FMT safer [20, 21]. At our institution, stool samples were obtained from "open biome", which is a standardized stool bank providing frozen fecal samples after screening for harmful pathogens (OpenBiome, n.d.). It will be interesting to follow patients for a longer period to determine 
the long-lasting effects of FMT on these patients. We followed patients in our study population for up to 2 months for symptom resolution and up to 1 year for recurrence and repeat FMT. Also, more studies are needed to analyze the effects of FMT on other diseases beyond CDI such as IBD, irritable bowel syndrome, metabolic syndrome, and non-alcoholic steatohepatitis $[22,23]$. Finally, measures should be taken to reduce the recurrence of CDI, and factors affecting recurrent disruption of colonic flora should be studied in more detail. The risk of symptomatic recurrence of CDI increases after every episode of CDI [24].

\section{Conclusions}

In conclusion, our single-center retrospective study showed that FMT is a safe and effective treatment option for patients with recurrent or severe CDI. Being on immunosuppressant medications correlates with the recurrence of CDI. The financial burden of CDI on hospitals may be minimized with frequent use of FMT by limiting the need for long-term use of expensive antibiotics and readmissions due to recurrent CDI. The use of frozen samples for FMT from a stool bank can ensure standardized screening. Universal screening and adoption of a single most effective FMT protocol can help determine the long-term safety and efficacy of FMT, especially in the immunosuppressed population.

\section{Acknowledgments}

The authors acknowledge administrative staff at the Department of Gastroenterology at Albany Medical Center for their help in this study.

\section{Financial Disclosure}

All authors declare that this study was not funded by a grant.

\section{Conflict of Interest}

The authors declare that there is no conflict of interest in this study.

\section{Informed Consent}

Not applicable

\section{Author Contributions}

MFA and AB conceived and designed the project. MFA, OT, and $\mathrm{YN}$ contributed to data collection. MFA and OT analyzed and interpreted the data. YN, MFA, and OT drafted the manuscript. All authors read and approved the final manuscript.

\section{Data Availability}

The authors declare that data supporting the findings of this study are available within the article.

\section{References}

1. Burden of clostridium difficile infection in the United States. N Engl J Med. 2015;372(24):2368-2370.

2. Lessa FC, Mu Y, Bamberg WM, Beldavs ZG, Dumyati GK, Dunn JR, Farley MM, et al. Burden of Clostridium difficile infection in the United States. N Engl J Med. 2015;372(9):825-834.

3. Khoruts A, Dicksved J, Jansson JK, Sadowsky MJ. Changes in the composition of the human fecal microbiome after bacteriotherapy for recurrent Clostridium difficile-associated diarrhea. J Clin Gastroenterol. 2010;44(5):354-360.

4. Guy PR. Coprophagy in the African elephant (Loxodonta Africana Blumenbach). Afr J Ecol. 1977;15(2).

5. Hopkins DW, Chudek JA, Bignell DE, Frouz J, Webster EA, Lawson T. Application of 13C NMR to investigate the transformations and biodegradation of organic materials by wood- and soil-feeding termites, and a coprophagous litterdwelling dipteran larva. Biodegradation. 1998;9(6):423431.

6. Zhang F, Luo W, Shi Y, Fan Z, Ji G. Should we standardize the 1,700-year-old fecal microbiota transplantation? Am J Gastroenterol. 2012;107(11):1755; author reply p 17551756.

7. de Groot PF, Frissen MN, de Clercq NC, Nieuwdorp M. Fecal microbiota transplantation in metabolic syndrome: History, present and future. Gut Microbes. 2017;8(3):253267.

8. Eiseman B, Silen W, Bascom GS, Kauvar AJ. Fecal enema as an adjunct in the treatment of pseudomembranous enterocolitis. Surgery. 1958;44(5):854-859.

9. McDonald LC, Gerding DN, Johnson S, Bakken JS, Carroll KC, Coffin SE, Dubberke ER, et al. Clinical practice guidelines for clostridium difficile infection in adults and children: 2017 update by the Infectious Diseases Society of America (IDSA) and Society for Healthcare Epidemiology of America (SHEA). Clin Infect Dis. 2018;66(7):e1-e48.

10. Lee CH, Steiner T, Petrof EO, Smieja M, Roscoe D, Nematallah A, Weese JS, et al. Frozen vs fresh fecal microbiota transplantation and clinical resolution of diarrhea in patients with recurrent Clostridium difficile infection: a randomized clinical trial. JAMA. 2016;315(2):142-149.

11. Surawicz CM, Brandt LJ, Binion DG, Ananthakrishnan AN, Curry SR, Gilligan PH, McFarland LV, et al. Guidelines for diagnosis, treatment, and prevention of Clostridium difficile infections. Am J Gastroenterol. 2013;108(4):478-498; quiz 499.

12. Borody T, et al. Fecal microbiota transplantation in ulcerative colitis: review of 24 years experience: 1644. Official Journal of the American College of Gastroenterology | ACG. 2012;107:S665.

13. Ramai D, Zakhia K, Ofosu A, Ofori E, Reddy M. Fecal 
microbiota transplantation: donor relation, fresh or frozen, delivery methods, cost-effectiveness. Ann Gastroenterol. 2019;32(1):30-38.

14. Shogbesan O, Poudel DR, Victor S, Jehangir A, Fadahunsi O, Shogbesan G, Donato A. A Systematic Review of the Efficacy and Safety of Fecal Microbiota Transplant for Clostridium difficile Infection in Immunocompromised $\mathrm{Pa}$ tients. Can J Gastroenterol Hepatol. 2018;2018:1394379.

15. Lubbert C, Johann C, Kekule AS, Worlitzsch D, Weis S, Mossner J, Behl S, et al. [Immunosuppressive treatment as a risk factor for the occurrence of clostridium difficile infection (CDI)]. Z Gastroenterol. 2013;51(11):1251-1258.

16. Trifan A, Stanciu C, Girleanu I, Stoica OC, Singeap AM, Maxim R, Chiriac SA, et al. Proton pump inhibitors therapy and risk of Clostridium difficile infection: Systematic review and meta-analysis. World J Gastroenterol. 2017;23(35):6500-6515.

17. Patil R, Blankenship L. Proton pump inhibitors and Clostridium difficile infection: are we propagating an already rapidly growing healthcare problem? Gastroenterology Res. 2013;6(5):171-173.

18. Linsky A, Gupta K, Lawler EV, Fonda JR, Hermos JA. Proton pump inhibitors and risk for recurrent Clostridium difficile infection. Arch Intern Med. 2010;170(9):772-778.

19. Mitchell BG, Gardner A. Mortality and Clostridium difficile infection: a review. Antimicrob Resist Infect Control. 2012;1(1):20.

20. Cammarota G, Ianiro G, Kelly CR, Mullish BH, Allegretti JR, Kassam Z, Putignani L, et al. International consensus conference on stool banking for faecal microbiota transplantation in clinical practice. Gut. 2019;68(12):21112121.

21. DeFilipp Z, Bloom PP, Torres Soto M, Mansour MK, Sater MRA, Huntley MH, Turbett S, et al. Drug-Resistant E. coli Bacteremia Transmitted by Fecal Microbiota Transplant. N Engl J Med. 2019;381(21):2043-2050.

22. Millan B, Laffin M, Madsen K. Fecal microbiota transplantation: beyond Clostridium difficile. Curr Infect Dis Rep. 2017;19(9):31.

23. Patel K, Patel A, Hawes D, Shah J, Shah K. Faecal microbiota transplantation: looking beyond clostridium difficile infection at inflammatory bowel disease. Gastroenterol Hepatol Bed Bench. 2018;11(1):1-8.

24. Lo Vecchio A, Cohen MB. Fecal microbiota transplantation for Clostridium difficile infection: benefits and barriers. Curr Opin Gastroenterol. 2014;30(1):47-53. 